\title{
Chiral criticality in helimagnet Ho studied by polarized neutron scattering
}

\author{
V. P. Plakhty, ${ }^{1,2}$ W. Schweika, ${ }^{2}$ Th. Brückel, ${ }^{2}$ J. Kulda, ${ }^{3}$ S. V. Gavrilov, ${ }^{1}$ L.-P. Regnault,${ }^{4}$ and D. Visser ${ }^{5}$ \\ ${ }^{1}$ Petersburg Nuclear Physics Institute, Gatchina, St. Petersburg 188300, Russia \\ ${ }^{2}$ Institut für Streumethoden, IFF, Forschungszentrum Jülich, D-52425 Jülich, Germany \\ ${ }^{3}$ Institut Laue-Langevin, Boîte Postale 156, 38042 Grenoble Cedex 9, France \\ ${ }^{4}$ CEA-Grenoble, DRFMC/SPSMS/MDN, rue des Martyrs, 38054 Grenoble Cedex 9, France \\ ${ }^{5}$ CLRC, ISIS Facility, Rutherford Appleton Laboratory, Chilton, Didcot OX11 OQX, United Kingdom
}

(Received 19 April 2001; published 8 August 2001)

\begin{abstract}
The critical exponents for the average chirality, $\beta_{c}=0.90(3)$, and the chiral susceptibility, $\gamma_{c}=0.68(6)$, are determined for the spin-ordering transition in helimagnet Ho. The $\beta_{c}$ is extraordinarily large and twice higher than the predicted value for the chiral universality class, while the $\gamma_{c}$ is in agreement with this scenario. The staggered magnetization critical exponent is determined to be $\beta=0.38(1)$. The difference $\beta_{c}-2 \beta$ $=0.137(36)$ suggests that chirality is a component of the order parameter independent of the staggered magnetization. The chiral-ordering transition and the Néel temperature coincide with a relative precision of $10^{-4}$.
\end{abstract}

DOI: 10.1103/PhysRevB.64.100402

PACS number(s): 75.25.+z, 75.40.Cx, 75.50.Ee

The order parameter of helically polarized magnets [stacked-triangular lattice antiferromagnets (TLA), helimagnets, such as rare-earth metals, etc.] includes, along with the ordinary spin variable $\mathbf{S}_{\mathbf{R}}$, the spin chirality

$$
\mathbf{C}=\left[\mathbf{S}_{\mathbf{R} 1} \times \mathbf{S}_{\mathbf{R} 2}\right]
$$

This is a relevant two-point variable that describes the clockwise or anticlockwise rotation between spins, $\mathbf{S}_{\mathbf{R} \mathbf{1}}$ and $\mathbf{S}_{\mathbf{R} \mathbf{2}}$, of nearest in-phase planes and is perpendicular to the wave vector $\mathbf{k}$ of the magnetic structure. A twofold additional degeneracy $\left(Z_{2}\right)$ changes the $\mathrm{SO}(2)$ symmetry of an $X Y$ magnet, two types of which are mentioned above, to $Z_{2}$ $\times \mathrm{SO}(2)$. In the case of a three-dimensional (3D)-spatial dimensionality, the universality class of the phase transition is basically determined by the number $n$ of the spin components that defines the anisotropy: easy axis Ising magnet ( $n$ $=1)$, easy-plane $X Y$ magnet $(n=2)$, and isotropic Heisenberg magnet $(n=3)$. The universality classes $O(n)$ of Wilson-Fisher are characterized by the critical exponents $\alpha$, $\beta, \gamma$, and $\nu$ for the specific heat, the order parameter, the susceptibility, and the inverse correlation length, respectively, as well as by the scaling relations and by the ratios of amplitudes above and below $T_{N}$ for the specific heat, $A^{+} / A^{-}$, and the susceptibility, $C^{+} / C^{-}$. A change of symmetry to $Z_{2} \times \mathrm{SO}(2)$ gives the notion of different universality classes. Kawamura ${ }^{1}$ formulated a hypothesis of new, chiral universality classes for the $X Y$ and Heisenberg TLA's on the basis of a symmetry analysis and Monte Carlo simulations. Nevertheless, this conjecture is strongly debated on the basis of Monte Carlo data ${ }^{2}$ and the nonlinear $\sigma$ model. $^{3}$ In any case, the chirality influences the critical behavior modifying the exponents $\alpha, \beta, \gamma$, and $\nu$. Besides, specific critical exponents, $\beta_{c}, \gamma_{c}$, and $\nu_{c}$ appear for the average chirality, the chiral susceptibility, and the chiral correlation length, respectively. The exponent $\beta_{c}$ is not necessarily equal to $2 \beta$ as one could naively expect from Eq. (1). (Reviews on the theoretical and experimental studies of the chiral systems can be found in Refs. 4 and 5.) We believe that one of the origins of the debates on the chiral universality classes is the fact that the chiral exponents have been directly measured only recently ${ }^{6}$ for a TLA $\mathrm{CsMnBr}_{3}$ using the polarized neutron scattering technique. Experimental values ${ }^{6}$ of the chiral critical exponents $\beta_{c}=0.44(2)$ and $\gamma_{c}=0.84(7)$ are in good agreement with the Monte Carlo results ${ }^{7} \beta_{c}=0.45(2)$ and $\gamma_{c}=0.77(5)$ for the chiral universality class of an $X Y$-type TLA. Together with the fact that spin and chiral ordering occur at the same temperature, and the scaling relation $\alpha$ $+2 \beta_{c}+\gamma_{c}=2.19(9)$ fulfills a renormalization-group value ${ }^{8}$ of 2 , provides evidence that the chiral-universality scenario is valid for the $X Y$ TLAs.

The experimental values of the conventional critical exponents for the rare-earth helimagnets $\mathrm{Ho}, \mathrm{Dy}, \mathrm{Tb}$ are very scattered, with the exception of the $\beta \approx 0.39 .^{4}$ The most pronounced for the $X Y$ chiral universality class is the specific heat exponent $\alpha$, which should be very $\operatorname{high}^{7,9}(\alpha$ $=0.34-0.40)$, in the case of Ho varies from $-0.02(1),{ }^{10}$ to $0.27(2) .{ }^{11}$ Two scales of fluctuations in the very vicinity of the phase transition ${ }^{12}$ further complicate the situation. The exponents $\gamma$ and $\nu$ are very different: $\gamma_{1}=1.24(15), \quad \nu_{1}$ $=0.55(4)$, and $\gamma_{2}=2-5, \nu_{2}=1.0(3)$ for the inverse correlation lengths of about $0.02 \AA^{-1}$ and $0.001-0.002 \AA^{-1}$, respectively. ${ }^{13}$ Existing data on conventional critical exponents would fit any universality class. Measurements of the chiral exponents could clear up the situation.

The $\beta_{c}$ exponent for the average chirality $\langle C\rangle$ can be obtained from the temperature behavior of the polarizationdependent part of the magnetic satellite intensity ${ }^{14}$ that is given by the second term in Eq. (2):

$$
\begin{aligned}
I(\mathbf{Q}) \propto & f^{2}(\mathbf{Q})\left\{\left\langle S_{z}\right\rangle^{2}\left[1+(\hat{\mathbf{Q}} \cdot \hat{\mathbf{C}})^{2}\right]\right. \\
& \left.+2\langle C\rangle(\hat{\mathbf{Q}} \cdot \hat{\mathbf{C}})\left(\hat{\mathbf{Q}} \cdot \mathbf{P}_{\mathbf{0}}\right)\left(n_{L}-n_{R}\right)\right\} \delta\left(\mathbf{Q}-\mathbf{b}_{\mathbf{m}}-\mathbf{k}\right),
\end{aligned}
$$

where $\mathbf{Q}$ is the scattering vector, $\hat{\mathbf{Q}}, \hat{\mathbf{C}}$ are unit vectors, $\mathbf{k}$ is the satellite wave vector of a reflection with the reciprocal lattice vector $\mathbf{b}_{\mathbf{m}}$, and $n_{L}, n_{R}$ are the populations of the leftand right-handed chiral domains, respectively. 


$$
\langle C\rangle \propto \Delta(\mathbf{Q})=I^{\uparrow}(\mathbf{Q})-I^{\downarrow}(\mathbf{Q}) \propto|\tau|^{\beta_{c}},
$$

where $I^{\uparrow}$ and $I^{\downarrow}$ are the intensities measured with $\mathbf{P}_{\mathbf{0}}$ parallel and antiparallel to $\mathbf{Q}$, and $\tau=\left(T-T_{N}\right) / T_{N}$ is the reduced temperature. The conventional critical exponent $\beta$ can be determined from the first term of Eq. (2) as

$$
\left\langle S_{z}\right\rangle^{2} \propto \Sigma(\mathbf{Q})=I^{\uparrow}(\mathbf{Q})+I^{\downarrow}(\mathbf{Q}) \propto|\tau|^{2 \beta} .
$$

With a known $\beta_{c}$, the critical exponent $\gamma_{c}$ for the chiral susceptibility can be found from the chiral crossover exponent $\phi_{c}=\beta_{c}+\gamma_{c}$. The latter enters the polarizationdependent completely inelastic part of the cross section that appears due to the dynamical chirality (DC) — the interaction between chiral fluctuations and a field-induced magnetization above $T_{N} .{ }^{15}$ This cross section is given by the difference of the energy spectra measured with the opposite neutron beam polarization, $\Delta(\epsilon)=I^{\uparrow}(\epsilon)+I^{\downarrow}(\epsilon)$, which has been shown ${ }^{16}$ to have at small $\epsilon$ the following form:

$$
\Delta(\epsilon)=A(\epsilon / \Gamma)\left[1+(\epsilon / \Gamma)^{2}\right]^{-2},
$$

where $A \propto \tau^{-\phi_{c}}$.

A crystal of holmium with a mosaicity of about $0.1^{\circ}$ was obtained by recrystallization. A cylinder of $3 \mathrm{~mm}$ in diameter and $3.5 \mathrm{~mm}$ long was cut off from the crystal by spark-cut erosion. The cylinder axis was oriented along the $\left[\begin{array}{lll}0 & 0 & 1\end{array}\right]$ direction. To measure the average chirality one needs a nonequal domain population (2), which has been created, as in Ref. 17, by the torsion deformation of about $2 \%$ around the cylinder axis that is collinear to the $\mathbf{C}$. For this purpose, the cylinder has been welded by electron beam to the tails of the distortion device made of polycrystalline holmium $15 \mathrm{~mm}$ in diameter. To avoid any scattering from the tails, they were painted with paint on the basis of gadolinium nitrate. The crystal in the torsion device was installed in the orange cryostat. The measurements of the Bragg intensities $I^{\uparrow}(\mathbf{k})$ and $I^{\downarrow}(\mathbf{k})$ of the first satellite, $\mathbf{b}_{\mathbf{m}}=0$, were carried out on the IN22 triple axis spectrometer at a thermal neutron guide of the ILL reactor. As the crystal was necessarily quite large, a highest available incident momentum of $k_{i}=4.1 \AA^{-1}$ was used to reduce extinction. The difference of $I^{\uparrow}(\mathbf{k})$ and $I^{\downarrow}(\mathbf{k})$ of about 3\% is seen in the inset to Fig. 1. This difference that is related to the difference of left- and right-handed domain populations was less than expected, but still high enough to provide sufficient statistics.

The temperature dependence of the peak intensity of a satellite $(00 \mu)$ belonging to $\mathbf{b}_{m}=\left(\begin{array}{lll}0 & 0 & 0\end{array}\right)$ has been measured in the range $120 \leqslant T(K) \leqslant 132$. First of all, a series of preliminary longitudinal $(\omega-2 \theta)$ and transverse $(\omega)$ scans were made to determine the temperature dependence of the maximum position, to estimate the Néel point and to measure the diffuse critical scattering above $T_{N}$. For the analysis of the final peak intensities we have considered the following systematic corrections: (i) critical diffuse scattering background underneath the Bragg reflection that is measured within our experimental resolution. For the difference of intensities this is particularly small, as compared to the sum. Note, according to Eq. (5) it should even vanish, while residuals seem to stem from the energy dependence for the acceptance; (ii)

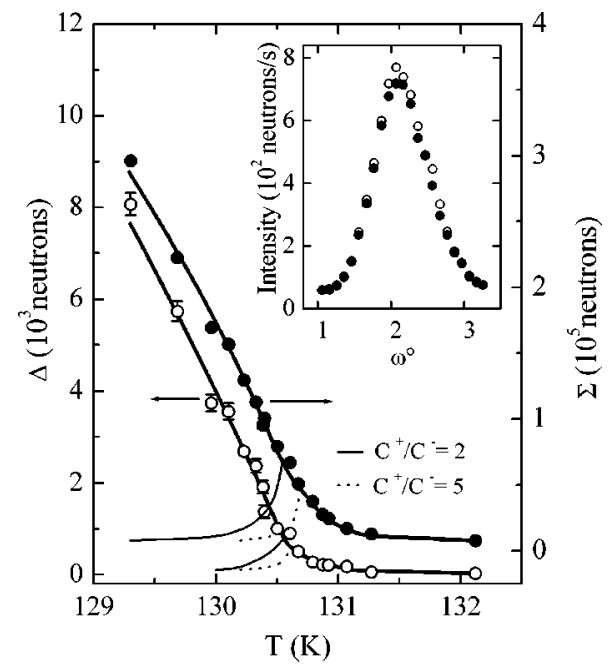

FIG. 1. Temperature dependence of the sum, $\Sigma$, and the difference, $\Delta$, of the peak intensities of the magnetic satellite $\left(\begin{array}{lll}0 & 0 \mu\end{array}\right)$ measured with the initial polarization parallel and antiparallel to the momentum transfer Q. Thin solid and dotted curves show possible corrections for the diffuse critical scattering. Rocking curves for the polarization parallel and antiparallel to $\mathrm{Q}$ are shown in the inset by the open and solid circles, respectively.

temperature dependence of the helix pitch, i.e., the angle between the spins in the vector product (1). But this correction turned out to be negligible in the temperature range studied; (iii) extinction effects, noticeable at low temperatures and farther away from the critical point, limiting the accessible critical region.

For correction (i) we can use the measured intensities above $T_{N}$ assuming a proper ratio $\left(C^{+} / C^{-}\right)$of the critical amplitudes. The limits of the ratio $C^{+} / C^{-}$are 2 for the mean field approximation and about 5 for the $\epsilon$-expansion and series (Table III in Ref. 18). The result ${ }^{19}$ for the ratio of the specific heat amplitudes $A^{+} / A^{-}=0.51(6)$ is close to the mean field value of 0.5 for a, $X Y$ system. (A different value ${ }^{11}$ is explained in Ref. 19 as a consequence of the data treatment with a discontinuous background at $T_{N}$, which contradicts renormalization-group studies.) Therefore, we have used a correction with $C^{+} / C^{-}=2$, as shown in Fig. 1 by the solid thin curves. To check the correction influence on the final results the calculations have been also made for the $C^{+} / C^{-}=5$.

The corresponding intensity correction is shown in Fig. 1 by the dotted curves. The data were treated as follows: At a temperature defined as a current Néel point $T_{N j}$ the corrections for the critical scattering were made, and the corrected values of the difference $\Delta(\tau)$ and sum $\Sigma(\tau)$ of the peak intensities were fit as $\log _{(10)} \Delta(\tau)=\log _{10} \Delta\left(\tau_{0}\right)+\beta_{c} \log _{10}|\tau|$ and $\log _{10} \Sigma(\tau)=\log _{10} \Sigma\left(\tau_{0}\right)+2 \beta \log _{10}|\tau|$ where $\tau_{0}$ was chosen to be 0.01 . Then the entire procedure was repeated at another $T_{N j}$. The final values of $T_{N}=130.55(2) K, \beta_{c}=0.79(3)$, and $2 \beta=0.67(1)$ have been chosen by the criteria of the residual least squares minimum, $\chi_{\min }^{2}=2.1$ for the $\Delta(\tau)$ and $\chi_{\min }^{2}=1.1$ for the $\Sigma(\tau)$. The results of the final iteration are shown in Fig. 2. The transition temperature for the chirality coincides with $T_{N}$, the spin ordering temperature, within the 


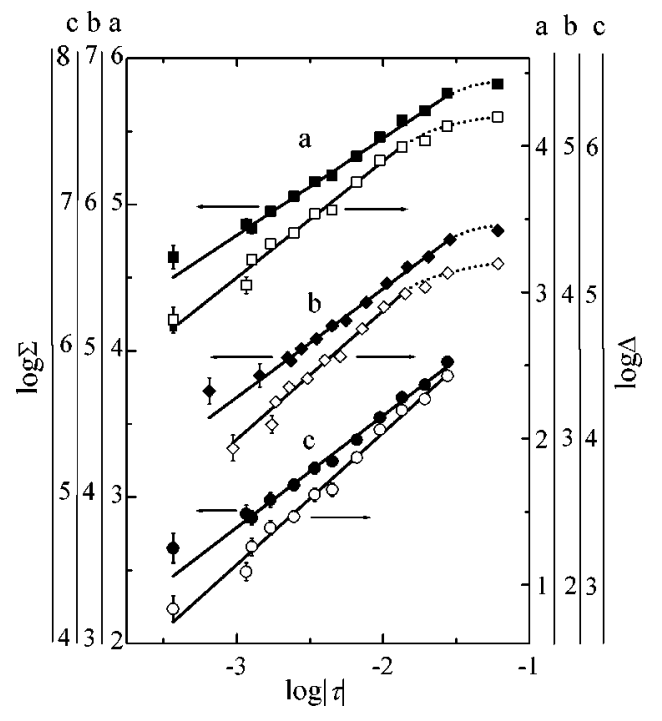

FIG. 2. The log-log plot of $\Sigma$ and $\Delta$ vs $\tau$. (a) and (b) Critical scattering correction with $C^{+} / C^{-}=2$ and $C^{+} / C^{-}=5$, respectively; (c) correction for the critical scattering with $C^{+} / C^{-}=2$, for the extinction and temperature dependence of the helix pitch.

limits of a standard deviation, i.e., a relative precision of $10^{-4}$. A similar procedure was used for the critical scattering correction with $C^{+} / C^{-}=5$. The exponents $\beta_{c}=0.88(4)$ and $2 \beta=0.74(2)$ have been obtained. In this case the chirality ordering temperature, $130.63(2) \mathrm{K}$, slightly differs from $T_{N}$ $=130.68$ (2) $K$, although not statistically significant. As seen from Fig. 2, there is a deviation from the straight lines at the highest reduced temperatures, which indicates a strong extinction in this region. The extinction correction in the range of $\tau$ used in the least squares refinement as shown for the ratio $C^{+} / C^{-}=2$ by the graphs under the label (c) has been made using the $\Sigma$ and $\Delta$-points for the highest $|\tau|=|\tau|_{\text {max }}$ in the following approximation: ${ }^{20}$

$$
I^{\uparrow}=I_{0}^{\uparrow}\left[1+x I_{0}^{\uparrow}\right]^{-1 / 2}, \quad I^{\downarrow}=I_{0}^{\downarrow}\left[1+x I_{0}^{\downarrow}\right]^{-1 / 2},
$$

where $I_{0}^{\uparrow}$ and $I_{0}^{\downarrow}$ are the intensities unperturbed by extinction with the opposite polarization, and $x$ is the extinction parameter. Taking for the unperturbed $\Sigma_{0}^{m}$ and $\Delta_{0}^{m}$ at $|\tau|=|\tau|_{\text {max }}$ the values obtained by extrapolation of $\log _{10} \Sigma(\tau)$ and $\log _{10} \Delta(\tau)$ to the $\log _{10}|\tau|_{\text {max }}$, one can obtain the extinction parameter $x$ from the equation:

$$
\Delta^{m}=\frac{\frac{1}{2}\left(\Sigma_{0}^{m}+\Delta_{0}^{m}\right)}{\sqrt{1+\frac{x}{2}\left(\Sigma_{0}^{m}+\Delta_{0}^{m}\right)}}-\frac{\frac{1}{2}\left(\Sigma_{0}^{m}-\Delta_{0}^{m}\right)}{\sqrt{1+\frac{x}{2}\left(\Sigma_{0}^{m}-\Delta_{0}^{m}\right)}}
$$

where $\Delta^{m}$ is the experimental difference at $|\tau|_{\text {max }}$. Then at any $|\tau|<|\tau|_{\text {max }}$ the extinction-free $\Sigma_{0}$ and $\Delta_{0}$ can be found from the measured values $\Sigma$ and $\Delta$ as

$$
\frac{\Sigma_{0} \pm \Delta_{0}}{(\Sigma \pm \Delta)^{2}}=\frac{x}{4}\left(1+\sqrt{1+\frac{16}{x^{2}(\Sigma \pm \Delta)^{2}}}\right) .
$$

TABLE I. Critical exponents $\beta_{c}, \beta$ obtained from the data corrected for the diffuse critical scattering: (a) $C^{+} / C^{-}=2$, (b) $C^{+} / C^{-}=5$, and (c) $C^{+} / C^{-}=2$ plus extinction correction.

\begin{tabular}{ccclc}
\hline \hline & $\beta_{c}$ & $\gamma_{c}$ & \multicolumn{1}{c}{$\beta$} & $\beta_{c}-2 \beta$ \\
\hline $\mathrm{a}$ & $0.794(29)$ & $0.83(5)$ & $0.334(7)$ & $0.125(32)$ \\
$\mathrm{b}$ & $0.880(32)$ & $0.70(5)$ & $0.372(8)$ & $0.136(36)$ \\
$\mathrm{c}$ & $0.901(28)$ & $0.68(5)$ & $0.382(11)$ & $0.137(36)$ \\
\hline \hline
\end{tabular}

The extinction influence on the $\Delta_{0}$ appears to be stronger than on $\Sigma_{0}$ as it follows from Eq. (8) and is seen in Fig. 2. The results are summarized in Table I together with $\gamma_{c}$ $=\phi_{c}-\beta_{c}$, where $\phi_{c}=1.58(4) .{ }^{21}$ As seen from the table the results are not very sensitive to the corrections. Nevertheless we believe that the most reliable result is represented in line (c). The arguments for the mean-field approximation $C^{+} / C^{-}=2$ have been given above.

The extinction correction noticeably changes the critical exponents. The final value of $\beta$ coincides in the limits of one standard deviation with $\beta=0.39(4)$ from a previous unpolarized neutron diffraction ${ }^{22,23}$ as well as with $\beta=0.37(10)$ for the narrow and broad peak components, with $\beta=0.39(4)$ in the bulk, $\beta=0.43(6)$ in the thin film, found from the resonance $\mathrm{x}$-ray scattering. ${ }^{13,24}$ The influence of the torsion on the $\beta$ value seems to be not essential as long as it is in good agreement with the data ${ }^{13,22-24}$ for the strain-free samples. Furthermore, the high value of $\beta_{c}$ should not be due to torsion since it is consistent with the high $\phi_{c}=\beta_{c}+\gamma_{c}$ obtained for the native crystal without deformation. ${ }^{21}$ There are neither $\beta$ nor $\beta_{c}$ calculated values for the chiral universality class of the $X Y$ helimagnets such as Ho, apparently due to the difficulties in including the long-range oscillating Ruderman-Kittel-Kasuya-Yosida (RKKY) interaction. Therefore the critical exponents are usually compared to those obtained for the $X Y$ TLA's. ${ }^{4}$ The $\beta$ exponent is essentially higher than $\beta=0.253(10)$ obtained by Monte Carlo simulation $^{7}$ or the experimental value ${ }^{25}$ of $0.21(2)$ for $\mathrm{CsMnBr}_{3}$. Moreover, the chiral critical exponent $\beta_{c}$ $=0.901(28)$ is twice as large in comparison with the Monte Carlo value ${ }^{7}$ of $\beta_{c}=0.45(2)$ and $\beta_{c}=0.44(2)$ obtained in the experiment ${ }^{6}$ for $\mathrm{CsMnBr}_{3}$.

Different from the situation studied in the Monte Carlo simulation, where the ordered ground state is characterized by a small finite number of spin orientations, in Ho the spin space is continuous in $x y$. This difference in symmetry of the ground state could be one origin for the different critical exponents and for another universality class. Apparently the ratio of the correlation length and the interaction scale that determines the helix pitch, or the dipolar interaction between the ferromagnetically ordered planes is important. ${ }^{19}$ This also holds for comparison with $\mathrm{CsMnBr}_{3}$. It is interesting that the chiral susceptibility exponent $\gamma_{c}=0.68(5)$, which characterizes the chirality fluctuations, is not too far from the corresponding Monte Carlo $^{7}$ and experimental ${ }^{6}$ values for a TLA $\mathrm{CsMnBr}_{3}, \gamma_{c}=0.77(5)$ and $\gamma_{c}=0.84(7)$, respectively. The $\beta$ exponent is in agreement with $\beta=0.39$ obtained in the 
theoretical work ${ }^{26}$ where the spin-ordering transition in Ho is described by the $\mathrm{O}(4)$ universality class, but it contains no data on the $\beta_{c}$ and $\gamma_{c}$.

As has been mentioned above, the exponent $\beta_{c}$ is not necessarily equal to $2 \beta$ as one could naively expect from Eq. (1). We have paid special attention to check whether $\beta_{c}$ $=2 \beta$. From Table I it is seen that independently of the correction used there is a stable difference of nearly four standard deviations, which is statistically significant. For the final result we take line (c) in Table I: $\beta_{c}-2 \beta=0.137$ (36) with a high reliability level. A possible explanation is that while $\beta$ describes the critical behavior of the longitudinal spin components $S_{z}, \beta_{c}$ refers also to the correlations in the transverse components, which enter the thermal average of the vector product (1). This difference means that the chirality is an independent component of the order parameter.

In summary we have measured the polarization-dependent and polarization-independent parts of the intensity of magnetic satellite from the Ho crystal under torsion in the reduced temperature range $4 \times 10^{-4} \leqslant \tau \leqslant 6 \times 10^{-2}$. The chiral critical exponents $\beta_{c}$ and $\gamma_{c}=\phi_{c}-\beta_{c}$, as well as a conventional exponent $\beta$ are determined. The $\beta_{c}$ is about two times higher than for the chiral transition in a TLA $\mathrm{CsMnBr}_{3}$, while the $\gamma_{c}$ differs little from the TLA chiral scenario value. Apparently, the critical behavior at the spin-ordering transition represents a crossover from the mean-field to the $X Y$ chiral universality scenario, ${ }^{4,27}$ as has been discussed above. To clear up the question on the universality class of the spinordering transition in helimagnets, a study of the chiral criticality in a helimagnetic insulator such as $\mathrm{MnO}_{2}$ is desirable. A difference between $\beta_{c}$ and $2 \beta$ is found, which suggests that the chirality infers an additional component of the order parameter independent of $S_{z}$.

We are indebted to S. V. Maleyev for fruitful discussions and O. P. Smirnov who found the possibility of the crystal cutting and welding. We acknowledge partial support by the Russian Foundation for Fundamental Researches (Grant Nos. 00-02-17273 and 00-15-96814), as well as by the Russian Ministry of Science (Contract No. 107-17-00). V. P. P. thanks FZ-Jülich for hospitality.
${ }^{1}$ H. Kawamura, J. Phys. Soc. Jpn. 54, 3220 (1985); 55, 2095 (1986); 56, 474 (1987); 58, 584 (1989).

${ }^{2}$ M. L. Plumer and A. Maihot, Phys. Rev. B 50, 16113 (1994).

${ }^{3}$ P. Azaria, B. Delamotte, and Th. Jolicoeur, Phys. Rev. Lett. 64, 3175 (1990).

${ }^{4}$ H. Kawamura, J. Phys. C 10, 4707 (1998).

${ }^{5}$ M. F. Collins and O. A. Petrenko, Can. J. Phys. 75, 605 (1997).

${ }^{6}$ V. P. Plakhty, J. Kulda, D. Visser, E. V. Moskvin, and J. Wosnitza, Phys. Rev. Lett. 85, 3942 (2000).

${ }^{7}$ H. Kawamura, J. Phys. Soc. Jpn. 61, 1299 (1992).

${ }^{8}$ H. Kawamura, Phys. Rev. B 38, 4916 (1988); J. Phys. Soc. Jpn. 59, 2305 (1990).

${ }^{9}$ R. Deutschmann, H. von Löhneysen, J. Wosnitza, R. K. Kremer, and D. Visser, Europhys. Lett. 17, 637 (1992).

${ }^{10}$ P. de Plessis, C. F. van Doorn, and D. C. van Delden, J. Magn. Magn. Mater. 40, 91 (1983).

${ }^{11}$ K. D. Jayasuriya, S. J. Campbell, and A. M. Stuart, J. Phys. F: Met. Phys. 15, 225 (1985).

${ }^{12}$ T. R. Thurston, G. Helgesen, Doon Gibbs, J. P. Hill, B. D. Gaulin, and G. Shirane, Phys. Rev. Lett. 70, 3151 (1993); Physica B 192, 177 (1993).

${ }^{13}$ T. R. Thurston, G. Helgesen, J. P. Hill, Doon Gibbs, B. D. Gaulin, and P. J. Simpson, Phys. Rev. B 49, 15730 (1994).

${ }^{14}$ S. V. Maleyev, V. G. Bar'yakhtar, and R. A. Suris, Fiz. Tverd. Tela (Leningrad) 4, 3461 (1962) [Sov. Phys. Solid State 4, 2533 (1963)]; Yu. A. Izyumov, Zh. Éksp. Teor. Fiz. 43, 1672 (1962)
[Sov. Phys. JETP 16, 1180 (1963)]; M. Blume, Phys. Rev. 130, 1670 (1963).

${ }^{15}$ S. V. Maleyev, Phys. Rev. Lett. 75, 4682 (1995).

${ }^{16}$ V. P. Plakhty, S. V. Maleyev, J. Kulda, J. Wosnitza, D. Visser, and E. Moskvin, Europhys. Lett. 48, 215 (1999).

${ }^{17}$ V. I. Fedorov, A. G. Gukasov, V. Kozlov, S. V. Maleyev, V. P. Plakhty, and I. A. Zobkalo, Phys. Lett. A 224, 372 (1997).

${ }^{18}$ E. Brezin, J. C. Le Guillou, and J. Zinn-Justin, in Phase Transitions and Critical Phenomena, Vol. 6, edited by C. Domb and M. S. Green (Academic Press, New York, 1976).

${ }^{19}$ J. Wang, D. P. Belanger, and B. D. Gaulin, Phys. Rev. Lett. 66, 3195 (1991).

${ }^{20}$ M. J. Cooper and K. D. Rouse, Acta Crystallogr., Sect. A: Found. Crystallogr. 26, 214 (1970).

${ }^{21}$ V. P. Plakhty, S. V. Maleyev, J. Kulda, D. Visser, J. Wosnitza, E. V. Moskvin, Th. Brückel, and R. K. Kremer, Physica B 297, 60 (2001).

${ }^{22}$ J. Eckert and G. Shirane, Solid State Commun. 19, 911 (1976).

${ }^{23}$ G. H. F. Brits and P. de V. du Plessis, J. Phys. F: Met. Phys. 18, 2659 (1988).

${ }^{24}$ G. Helgesen, J. P. Hill, T. R. Thurston, Doon Gibbs, J. Kwo, and M. Hong, Phys. Rev. B 50, 2990 (1994).

${ }^{25}$ T. E. Mason, B. D. Gaulin, and M. F. Collins, Phys. Rev. B 39, 586 (1989).

${ }^{26}$ P. Bak and D. Mukamel, Phys. Rev. B 13, 5086 (1976).

${ }^{27}$ J. Wang, D. P. Belanger, and B. D. Gaulin, Phys. Rev. Lett. 66, 3195 (1991). 